\title{
Refractory Chest Pain or Treating Cardiologist's Bane: A Case Report
}

\author{
Anupama V. Hegde ${ }^{1}$ Abhinay Tibdewal ${ }^{1}$ Vadagenalli S. Prakash ${ }^{1}$ Sarthak Sahoo ${ }^{1}$ \\ ${ }^{1}$ Department of Cardiology, M. S. Ramaiah Medical College and \\ Hospitals, Bengaluru, Karnataka, India \\ Indian J Cardiovasc Dis Women-WINCARS 2017;2:49-53. \\ Address for correspondence Anupama V. Hegde, MBBS, DNB \\ (Medicine), DNB (Cardiology), Department of Cardiology, M. S. \\ Ramaiah Medical College and Hospitals, Bengaluru 560054, \\ Karnataka, India (e-mail: anupamadnb@rediffmail.com).
}

\begin{abstract}
Keywords

- aortic valve replacement

- coronary angiography

- microvascular angina

- refractory chest pain

Microvascular angina is caused by dysfunction of small-resistance coronary arteries $(<500 \mu \mathrm{m})$ and is of heterogenous origin. The major epicardial coronaries are normal and commonly seen in women. Prognosis is variable, with disabling angina in many patients and can be a cause of mortality, especially in those who are refractory to treatment. In this background, we present a case of 56-year-old post valve replacement with normally functioning aortic valve and recurrent episodes of microvascular angina. The patient had normal epicardial coronaries. She had recurrent episodes of angina refractory to various antianginals associated with hemodynamic instability. Microvascular angina can curtail routine activity, frequent hospitalization, and repeated noninvasive and invasive investigations. Thus, it is a high social and economical disease, especially pertinent to women.
\end{abstract}

\section{Introduction}

The first mention of chest pain with normal coronaries was made in 1981 by Opherk and coworkers. ${ }^{1}$ Among patients undergoing coronary angiography (CAG) for ischemic signs and symptoms, approximately 10 to $30 \%$ have angiographically normal coronaries. Coronary microvascular dysfunction is present in 50 to $60 \%$ of this subgroup. The scenario is different from that of coronary vasospasm, where major coronary arteries are affected with either complete occlusion with or without collateral flow, or incomplete occlusion as evidenced by electrocardiographic (ECG) changes. Angina which is caused secondary to ischemia related to dysfunction of small resistance coronary artery vessels $(<500 \mu \mathrm{m})$ not visible in angiogram, is called "micro vascular angina." The pathophysiology of chest pain in women without obstructive coronary disease represents a heterogeneous mix: some have cardiac chest pain that is nonischemic, others have cardiac chest pain due to microvascular ischemia or abnormal pain sensation, and still others have chest pain of noncardiac origin. All these women suffer from symptoms of chest pain; however, the prognosis and therapeutic options differ widely. ${ }^{3}$ In 1973 , Kemp et al introduced the term "syndrome X" (later referred as "cardiac syndrome X") to describe patients with exercise- induced angina and normal coronary angiograms. However, the use of this term has not always been limited to this specific meaning. ${ }^{4}$

\section{Case Report}

A 56-year-old female patient presented with a history of dyspnea and angina of acute onset with no other cardiac symptoms. The patient had undergone aortic valve replacement with a mechanical tilting disc prosthetic valve 13 years ago for rheumatic aortic stenosis. She was on oral anticoagulation for the same and on oral hypoglycemic drugs for her diabetes. On evaluation, she was hemodynamically stable, afebrile, and auscultation revealed prosthetic valve click. ECG showed sinus rhythm with ST-T flattening in inferior leads with deep T-wave inversions in precordial leads. Troponin I was strongly positive. Blood chemistry including lipid profile was within normal limits. Two-dimensional (2D) transthoracic echocardiogram (TTE) revealed a peak gradient/mean gradient $-47 / 23 \mathrm{~mm} \mathrm{Hg}$ across the aortic prosthesis, normally functioning valve, normal left ventricular (LV) systolic function and grade II LV diastolic dysfunction, no regional wall-motion abnormality and normal pulmonary artery pressures, and no pericardial effusion/ 

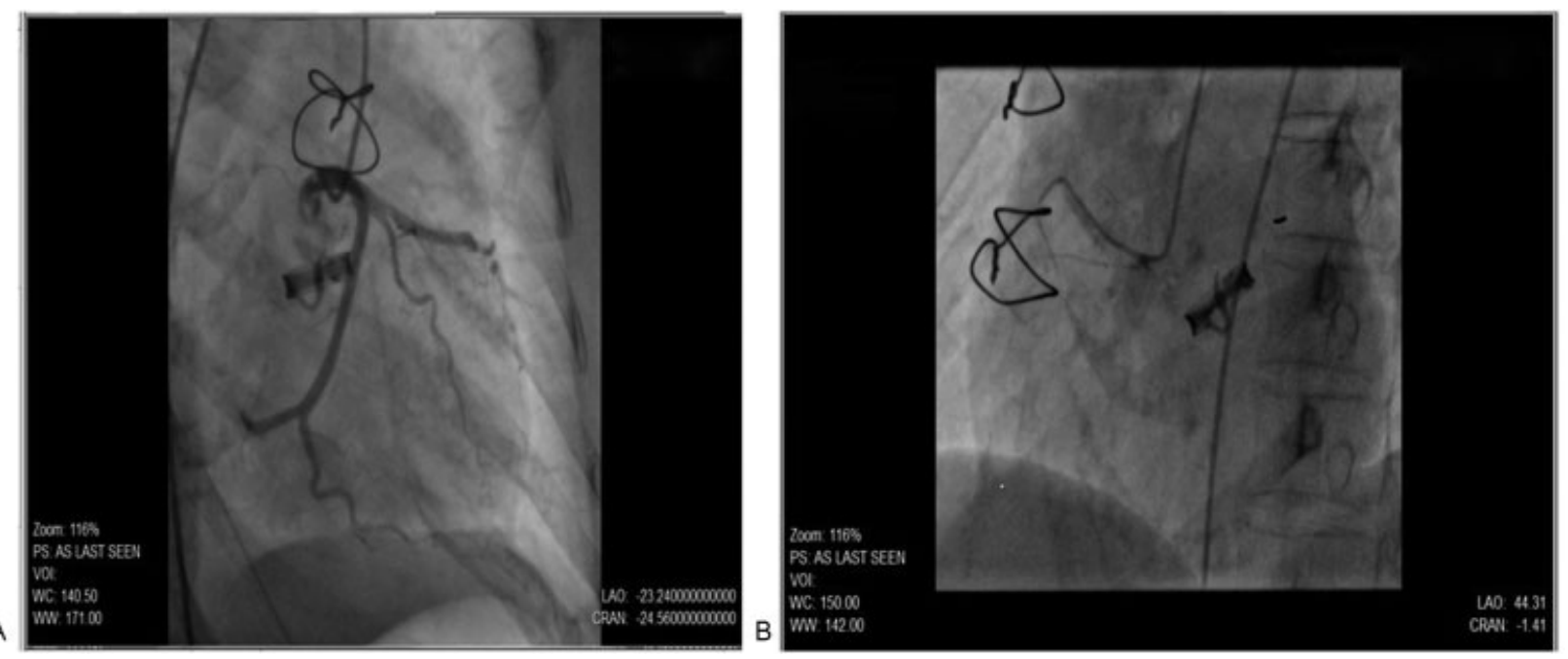

Fig. 1 (A, B) Normal coronaries on coronary angiography

clots/vegetation. The international normalized ratio (INR) was 1.9. Coronary angiogram revealed normal epicardial coronaries (-Fig. 1A, B).

The next day, the patient had a recurrent episode of angina with hemodynamic instability, requiring inotropic support. The episode lasted for an hour during which, the patient was hypotensive and developed deep ST depression in all leads except for ST elevation in aVR and V1 (-Fig. 2).

The angina responded to IV nicorandil. With resolution of angina, the patient stabilized and ECG changes settled (-Fig. 3). For comparison, basal ECG is mentioned in -Fig. 4.
Subsequently, the patient had multiple similar episodes of angina with similar ECG changes as described previously, and repeat echo did not reveal any fresh abnormality. Fluoroscopy revealed a normally functioning tilting disc prosthetic valve. In view of ECG changes, repeat CAG was done (during angina episode), which again showed normal coronaries and absence of coronary vasospasm (-Fig. 5).

Serum analysis revealed normal electrolytes and normal serum calcium. Over the next 5 days, the patient had multiple episodes of angina that responded transiently to IV infusion of nicorandil and she was continued on maximum

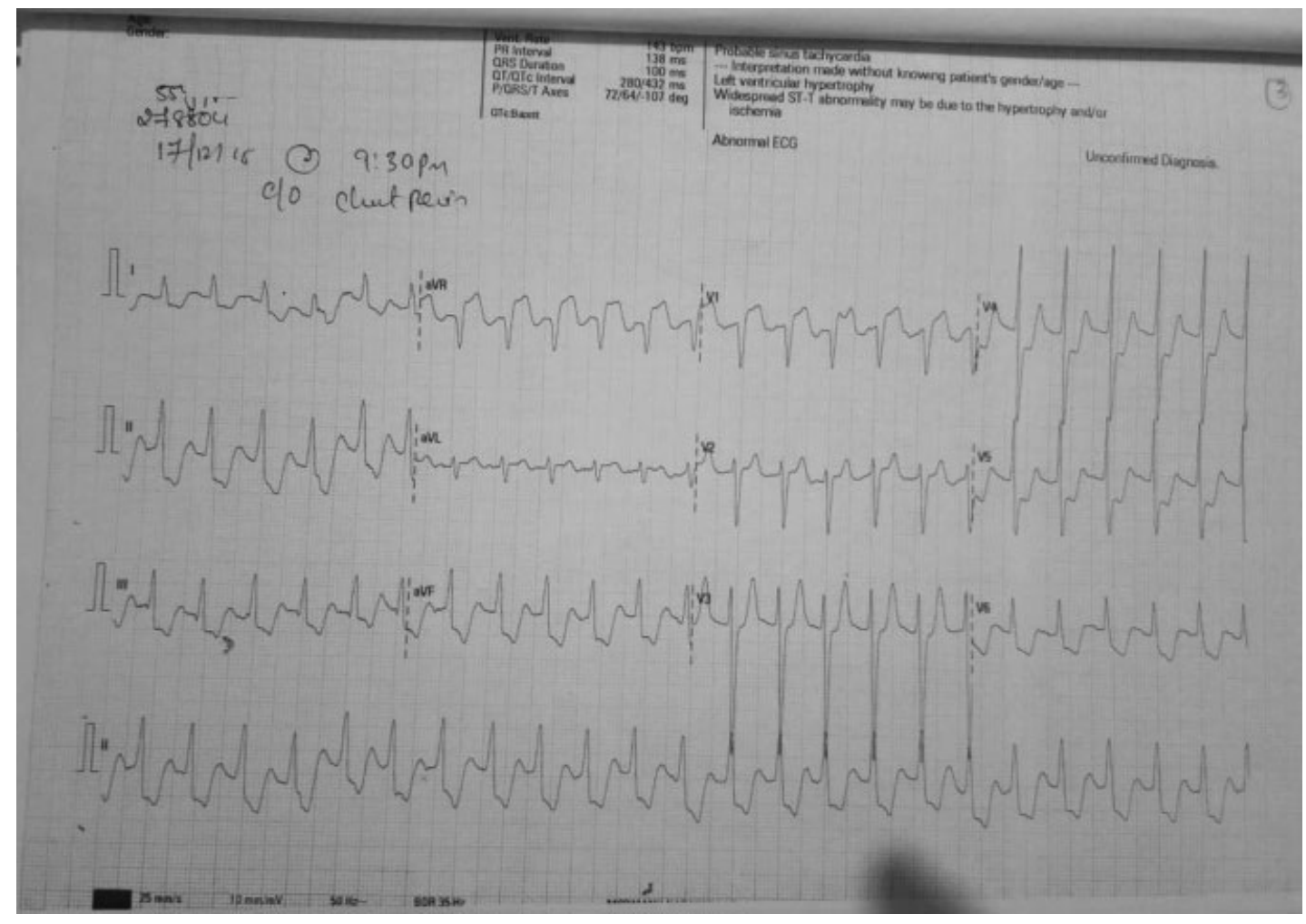

Fig. 2 Electrocardiography during angina. 


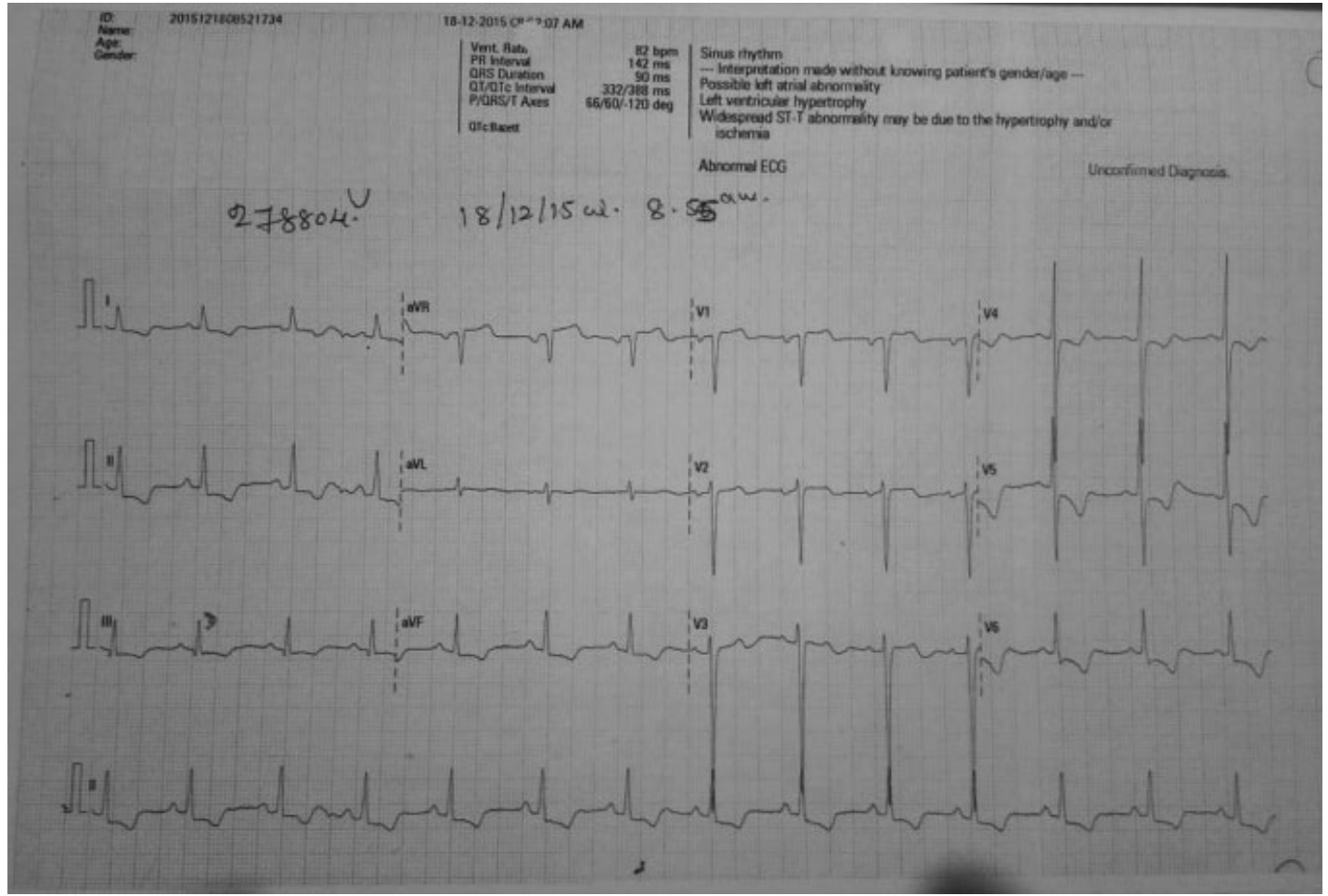

Fig. 3 Electrocardiography after resolution of angina.

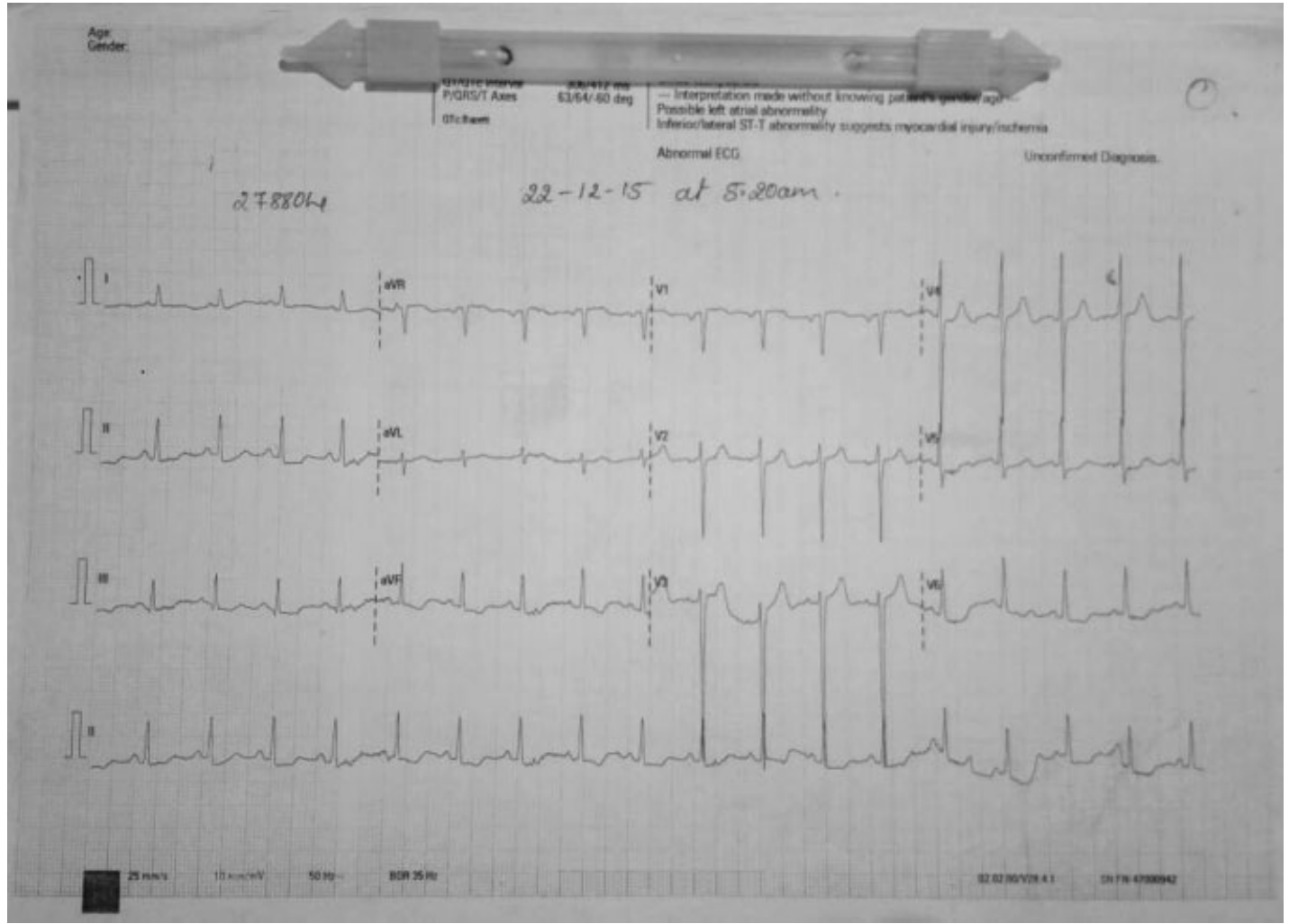

Fig. 4 Basal electrocardiography. 


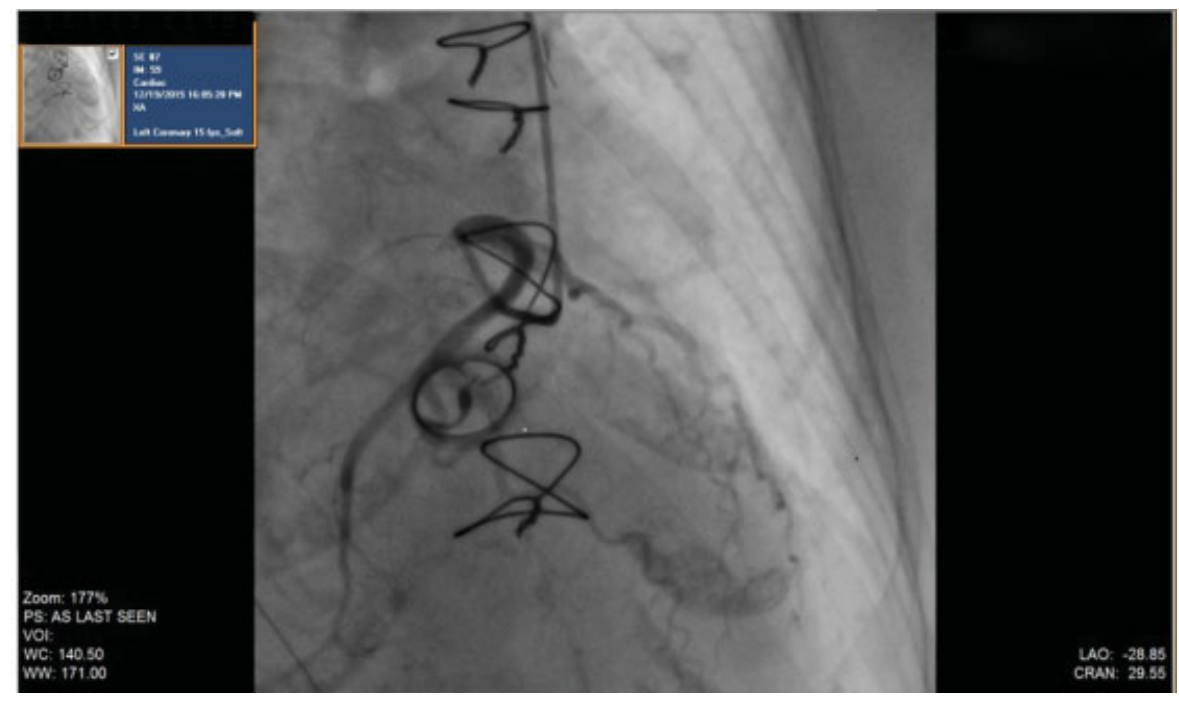

Fig. 5 Coronary angiography during angina.

antianginal therapy. She was also initiated on endothelin receptor antagonist Ambrisentan, but the response was uncertain. She continued to have angina episodes with hypotension. Incidentally she developed dense hemiplegia of the right side and was found to have labile INR with INR value of 7.1. Computed tomography (CT) of the brain showed intracerebral bleed. The patient gradually deteriorated, developed respiratory distress, and was put on ventilator. However, she did not improve and eventually succumbed.

\section{Discussion}

Angina without coronary artery disease (CAD) has substantial morbidity. Data regarding mortality in such cases are not clear as also is the management protocol. This case is being reported as it was a challenge to formulate a treatment plan for this patient with recurrent episodes of severe rest angina with hemodynamic instability and near-normal intervals during which she was completely asymptomatic and stable. The significant ECG changes along with normal coronaries demonstrated on CAG twice and normally functioning prosthetic valve with positive transient response to nitrates and nicorandil led us to a diagnosis of microvascular angina or syndrome $\mathrm{X}$. Provocation tests and imaging diagnostic modalities were not used as the patient was hemodynamically unstable. ECG changes in microvascular angina include ST-segment depression during episodes of spontaneous or provoked angina ${ }^{5}$ in contrast to vasospastic angina, where patients develop ST elevation and/or depression during chest pain episodes. Other changes include peaking and/or increase in amplitude of the T wave, a delay in the peak and an increase in the height and width of the $\mathrm{R}$ wave resulting in fusion of $\mathrm{R}$ wave with T wave, and a decrease in magnitude or disappearance of the $S$ wave. The negative $U$ wave may also appear at the beginning or near the end of the attack, and it is often associated with ST-segment changes in the anterolateral leads. ${ }^{6}$

In spite of therapy with all antianginals including nitrates, calcium channel blockers (CCBs), endothelin receptor antago- nists (ERAs), nicorandil, ranolazine, trimetazidine, and adequate sedation on a background therapy with aspirin $75 \mathrm{mg}$, statins, $\beta$ blockers, angiotensin-converting enzyme (ACE) inhibitors, and ivabradine, the patient continued to have recurrent angina. This definitely suggests a multifactorial pathogenesis for microvascular angina not responding to standard antianginal therapy. Two prevailing hypotheses have emerged to explain this phenomenon: 3,7 the ischemic hypothesis suggesting abnormal coronary microvascular function and the nonischemic hypothesis describing mainly altered pain perception and myocardial hypersensitivity. The amalgamation of increased demand and reduced vasodilator reserve may definitely promote the progress of a mismatch not severe enough to cause extensive myocardial ischemia and yet enough to induce delicate variations of various cardiac functions. A commonly reported aberration is endothelial dysfunction advocated by a decreased coronary flow response to acetylcholine, atrial pacing, and other endothelium mediated vasodilator stimuli, and believed to be caused by impaired nitric oxide (NO) release and/or activity. ${ }^{8}$

Given its unique pathophysiology and prognosis, it is imperative that microvascular angina be considered independently of other causes of chest pain. The current gold standards for evaluating microvascular function have been coronary flow reserve (CFR) using invasive testing and myocardial perfusion reserve (MPR) using positron emission tomography (PET) or cardiac magnetic resonance imaging (CMRI) analysis. After reviewing literature, we found that there is little substantiation to provide current treatment strategies for tangibly defined microvascular angina.

The standard practice of using antianginals and risk reduction modalities may not be effective in such patients and actually might be counterproductive in certain cases. Treatments with renin angiotensin aldosterone system (RAAS) inhibitors, ${ }^{9}$ CCBs, ${ }^{10}$ and statins have showed no substantial improvement in symptomatology. There was no significant difference in CFR measurements in patients with CFR $>2.5$ in a study with sildenafil and the effect on symptoms was not evaluated. ${ }^{11}$ L-Arginine; a precursor of NO, showed no improvement in 
symptoms after infusion. ${ }^{12}$ Neither $\alpha$-blockers, such as doxazosin, nor estrogen therapy were useful. $\beta$-Blockers decrease myocardial oxygen demand and increase coronary diastolic perfusion; hence, they have a convincing possible role in the treatment of microvascular angina, but studies supporting its role are lacking. Similarly, the newer antianginals such as ranolazine, trimetazidine, and nicorandil have not shown a sustained benefit, although in our case the patient did respond briefly to an infusion of nicorandil. Several supplementary therapies such as adenosine receptor blockers, tricyclic antidepressants, aerobic exercises, metformin, vitamin $\mathrm{C}$, anticoagulants, cognac, and traditional Chinese medical techniques have been evaluated with no success. Anecdotal reports suggest a probable role of endothelin-1 receptor antagonists in management of refractory microvascular angina though definitive results are not available. ${ }^{13,14}$

Numerous patients with angina and normal coronary arteries show persistence and even deterioration of angina over time. The episodes may become more regular, protracted, poorly responsive to drugs, and may significantly curtail routine activities. Moreover, the severity of angina may lead to frequent noninvasive testing, frequent hospital admissions for repeat CAG, and also frequent absence or even retirement from work. Thus, in these patients quality of life is often severely compromised. ${ }^{5}$ These traits make microvascular angina a socially and economically pertinent cardiac disease, especially in women. This emphasizes the urgent need to formulate a standard and easily available diagnostic and treatment protocol as well as encourage more research into this chest pain with "nos dimensions" and also the fact that therapy needs to be tailor made and structured according to individual patient profile.

\section{References}

1 Opherk D, Zebe H, Weihe E, et al. Reduced coronary dilatory capacity and ultrastructural changes of the myocardium in patients with angina pectoris but normal coronary arteriograms. Circulation 1981;63(04):817-825

2 Cannon RO III, Epstein SE. "Microvascular angina" as a cause of chest pain with angiographically normal coronary arteries. Am J Cardiol 1988;61(15):1338-1343

3 Bugiardini R. Normal coronary arteries: clinical implications and further classification. Herz 2005;30(01):3-7

4 Kemp HG Jr, Vokonas PS, Cohn PF, Gorlin R. The anginal syndrome associated with normal coronary arteriograms. Report of a six year experience. Am J Med 1973;54(06):735-742

5 Crea F, Lanza GA. Angina pectoris and normal coronary arteries: cardiac syndrome X. Heart 2004;90(04):457-463

6 Yasue H, Nakagawa H, Itoh T, Harada E, Mizuno Y. Coronary artery spasm-clinical features, diagnosis, pathogenesis, and treatment. J Cardiol 2008;51(01):2-17

7 Melikian N, De Bruyne B, Fearon WF, MacCarthy PA. The pathophysiology and clinical course of the normal coronary angina syndrome (cardiac syndrome X). Prog Cardiovasc Dis 2008;50(04):294-310

8 Chen JW, Hsu NW, Wu TC, Lin SJ, Chang MS. Long-term angiotensin-converting enzyme inhibition reduces plasma asymmetric dimethylarginine and improves endothelial nitric oxide bioavailability and coronary microvascular function in patients with syndrome X. Am J Cardiol 2002;90(09):974-982

9 Bavry AA, Handberg EM, Huo T, et al. Aldosterone inhibition and coronary endothelial function in women without obstructive coronary artery disease: an ancillary study of the national heart, lung, and blood institute-sponsored women's ischemia syndrome evaluation. Am Heart J 2014;167(06):826-832

10 Sütsch G, Oechslin E, Mayer I, Hess OM. Effect of diltiazem on coronary flow reserve in patients with microvascular angina. Int J Cardiol 1995;52(02):135-143

11 Denardo SJ, Wen X, Handberg EM, et al. Effect of phosphodiesterase type 5 inhibition on microvascular coronary dysfunction in women: a Women's Ischemia Syndrome Evaluation (WISE) ancillary study. Clin Cardiol 2011;34(08):483-487

12 Bøttcher M, Bøtker HE, Sonne H, Nielsen TT, Czernin J. Endothelium-dependent and -independent perfusion reserve and the effect of L-arginine on myocardial perfusion in patients with syndrome X. Circulation 1999;99(14):1795-1801

13 de Lemos J, Omland T. Chronic coronary artery disease: a companion to Braunwald's heart disease E-book. Cardiology 2018

14 Mittal SR. Management options for coronary microvascular dysfunction: present status and future perspectives. J Clin Prev Cardiol 2016;5(01):18-24 